\title{
TIME DISCRETIZATION OF NONLINEAR CAUCHY PROBLEMS APPLYING TO MIXED HYPERBOLIC-PARABOLIC EQUATIONS
}

\author{
PIERLUIGI COLLI \\ Dipartimento di Matematica, Università di Torino \\ Via Carlo Alberto 10, 10123 Torino, ITALY \\ ANGELO FAVINI \\ Dipartimento di Matematica, Università di Bologna \\ Piazza di Porta San Donato 5, 40127 Bologna, ITALY
}

(Received March 2, 1994 and in revised form April 4, 1995)

\begin{abstract}
In this paper we deal with the equation $L\left(d^{2} u / d t^{2}\right)+B(d u / d t)+A u \ni f$, where $L$ and $A$ are linear positive selfadjoint operators in a Hilbert space $H$ and from a Hilbert space $V \subset H$ to its dual space $V^{\prime}$, respectively, and $B$ is a maximal monotone operator from $V$ to $V^{\prime}$. By assuming some coerciveness on $L+B$ and $A$, we state the existence and uniqueness of the solution for the corresponding initial value problem. An approximation via finite differences in time is provided and convergence results along with error estimates are presented.
\end{abstract}

KEY WORDS AND PHRASES. Nonlinear second-order evolution equations, Cauchy problem, existence and uniqueness, time discretization, convergence and error estimate.

1991 AMS SUBJECT CLASSIFICATION CODES. 35M10, 35A05, 35A35.

\section{INTRODUCTION.}

Consider two Hilbert spaces $V$ and $H$ such that, by identifying $H$ with its dual space $H^{\prime}$, $V \subset H \subset V^{\prime}$ with dense and continuous injections. Let $L: H \rightarrow H$ and $A: V \rightarrow V^{\prime}$ be two linear, bounded, and selfadjoint operators. In addition, it is assumed that $L$ and $A$ are monotone, that is, non negative due to their linearity, and that $A$ satisfies some condition of coerciveness. Also, let $B$ be a maximal monotone operator from $V$ to $V^{\prime}$, possibly nonlinear and multivalued. Then, the present paper is concerned with the Cauchy problem for evolution equations of the form

$$
\begin{gathered}
\left.L \frac{d^{2} u}{d t^{2}}+B \frac{d u}{d t}+A u \ni f \quad \text { in }\right] 0, T[ \\
u(0)=u_{0}, \quad \frac{d u}{d t}(0)=v_{0}
\end{gathered}
$$

where $T>0, f:] 0, T\left[\rightarrow V^{\prime}\right.$ and $u_{0}, v_{0} \in V$ are given. In the case where $L$ coincides with the identity operator in $H$, the problem (1.1-2) is well-known. The existence and uniqueness of the solution has been originally established by Lions and Strauss [9] by using a Faedo-Galerkin 
approximation procedure. Another simple proof has been given by Brézis [3] (cf. also [1, Chapter V]) in the framework of the theory of nonlinear semigroups. It should be remarked that such a result applies to various significant cases of hyperbolic partial differential equations. For applications we refer to $[1,8]$ and especially to [9], where other hyperbolic problems, not included in this setting, are considered.

Here, we are interested to investigate (1.1-2) when $L$ is possibly degenerate, provided that the sum $L+B$ be coercive in $H$. Thus, our analysis concerns initial-boundary value problems for partial differential equations and systems, nonlinear in the first time derivative, of mixed hyperbolic-parabolic type. The hypothesis of coerciveness for $L+B$ implies that, in the set where degeneration of $L$ may occur, the operator $B$ does not degenerate, and conversely. Also the linear mapping $A$ is required to be coercive, but with respect to the space $V$ in a weak sense (cf. the later assumption (2.5)), following a usual position (see, e.g., [1, Chapter V]). Examples of operators $L, A$, and even $B$ can be constructed very easily, for instance when $H=\left(L^{2}(\Omega)\right)^{M}$ and $V=\left(H^{1}(\Omega)\right)^{M}(M \geq 1), \Omega$ denoting a bounded domain of $\mathbf{R}^{N}(N \geq 1)$. Regarding this matter, the reader can find several interesting choices still in $[9,1]$ or in $[2,11]$ as well. Let us just point out that, while the selfadjoint operators $L$ and $A$ are obviously subgradients of convex (and quadratic) functions, here $B$ is merely assumed to result maximal monotone, then not necessarily cyclically monotone. This allows to consider a wide range of applications, especially to systems of equations (it suffices to take into account the case of linear maps from $\mathbf{R}^{M}$ to $\mathbf{R}^{M}$ with $M>1$ ). Two model examples of partial differential equations are proposed in Section 2.

By reducing $B$ to be a linear and bounded operator in $H$, the resulting initial value problem for (1.1) has been treated by Bensoussan, Lions, and Papanicolau [2], who stated the existence and uniqueness of a weak solution. Further, some extensions of this result have been given in $[11,7,10]$ for a nonlinear right hand side $f(u)$ and for operators $L, B$ possibly depending on time. However, a careful analysis of the linear case (already addressed in [5, Chapter 3]) is carried out in [6], where strong solutions of (1.1) are examined and discussed under rather general conditions on the mapping $B$.

In this paper we seek solutions of (1.1) with first derivative in $L^{\infty}(0, T ; V)$ and second derivative in $L^{2}(0, T ; H)$ so that the initial conditions (1.2) make sense (apparently, one should instead specify the initial values of $u$ and $L(d u / d t))$. It is worth emphasizing that, consequently, our solutions are not really weak solutions. The question whether or not, under our framework, one can give weaker solutions to (1.1) remains open, even though it seems difficult (to find them) having to face with the nonlinearity in $d u / d t$. However, a partial answer is known for the reduced equation

$$
\left.\frac{d}{d t}\left(L \frac{d u}{d t}\right)+B \frac{d u}{d t}+A u \ni f \quad \text { in }\right] 0, T[
$$

investigated by Carroll and Showalter [5, Theorem 6.18 and Remark 6.19, pp. 223-224] when $B$ is monotone and hemicontinuous and $A, L$ are symmetric continuous linear operators from $V$ to $V^{\prime}$, with $L$ monotone and $A$ coercive. An existence and uniqueness theorem is derived for the related Cauchy problem by tranforming (1.3) into a first order system in a suitable product space, namely $V^{\prime} \times V_{L}^{\prime}$ (where $V_{L}^{\prime}$ is the dual of $V$ endowed with the seminorm $\langle L v, v\rangle^{1 / 2}, v \in V$ ). Actually, the solution to (1.3) discussed in [5] is weak since $u \in W^{1,1}(0, T ; V), B(d u / d t) \in$ $W^{1,1}\left(0, T ; V_{L}^{\prime}\right)$, and nothing can be said on the regularity of the second derivative of $u$.

The objective of our work is not only to establish existence and uniqueness properties for the solutions of (1.1-2), but also to introduce a discrete approximation of the problem, then prove convergence of the discrete solution to the continuous one, and finally estimate their difference. 
Thus, after providing a precise variational setting for (1.1-2) and showing the uniqueness result (see Section 2), in Section 3 we discretize the problem by a backward finite differences scheme. Next, a priori estimates are demonstrated in Section 4 and allow us to pass to the limit in Section 5, hence obtaining both the existence of a continuous solution and the convergence of the discrete one. The concluding Section 6 is devoted to the deduction of error estimates with respect to the time step of discretization.

\section{FORMULATION OF THE CAUCHY PROBLEM.}

First, we recall the basic notations and state precise assumptions on operators and data. Fix some number $T>0$ and let the real Hilbert space $V$ and $H$ satisfy $V \subset H$ with dense and continuous embedding. We identify $H$ with its dual space and denote by $V^{\prime} \supset H$ the dual space of $V$. Henceforth the notation $(\cdot, \cdot)$ will represent either the scalar product in $H$ or the duality pairing between $V^{\prime}$ and $V$. For the sake of simplicity, the norms in $V, H, V^{\prime}$ will be indicated by $\|\cdot\|,|\cdot|,\|\cdot\|_{*}$, respectively.

We introduce two bilinear symmetric forms $\ell: H \times H \rightarrow \mathbf{R}$ and $a: V \times V \rightarrow \mathbf{R}$ satisfying

$$
\begin{gathered}
\exists C_{\ell}>0: \quad|\ell(v, z)| \leq C_{\ell}|v||z| \quad \forall v, z \in H, \\
\quad \ell(v, v) \geq 0 \quad \forall v \in H, \\
\exists C_{a}>0: \quad|a(v, z)| \leq C_{a}\|v\|\|z\| \quad \forall v, z \in V,
\end{gathered}
$$

and, with the position

$$
a_{\alpha}(v, z):=a(v, z)+\alpha(v, z) \quad \forall \alpha \in \mathbf{R}, \forall v, z \in V
$$

fulfilling

$$
\forall \alpha>0 \quad \exists \omega_{\alpha}>0: a_{\alpha}(v, v) \geq \omega_{\alpha}\|v\|^{2} \quad \forall v \in V .
$$

Hence, it results that the operator $L: H \rightarrow H$ associated with the form $\ell$, that is

$$
(L v, z)=\ell(v, z) \quad \forall v, z \in H
$$

is continuous and maximal monotone. Moreover, as $\ell$ is symmetric, $L$ is selfadjoint and coincides with the subdifferential of the convex and continuous function

$$
\varphi(v)=\ell(v, v) / 2, \quad v \in H
$$

We denote by $\Lambda$ the square root of the operator $L$, so that

$$
(\Lambda v, \Lambda z)=\ell(v, z) \quad \forall v, z \in H
$$

It is straightforward to infer that $\Lambda: H \rightarrow H$ is continuous and selfadjoint as well. Next, let $A: V \rightarrow V^{\prime}$ be the bounded linear selfadjoint operator defined by

$$
(A v, z)=a(v, z) \quad \forall v, z \in V \text {. }
$$

Then, (2.5) turns out to be a coerciveness property for $A$. Indeed, from (2.5) it follows that for any $\alpha>0$ the mapping $\alpha I+A$ ( $I$ denoting the identity in $H$ ) is strongly monotone from $V$ to $V^{\prime}$. 
As already stated in the Introduction, for the operator $B$ we assume that

$$
B \text { is maximal monotone from } V \text { to } V^{\prime} \text {, }
$$

with domain $D(B)$. Also, it is required that the sum $L+B$ is strongly monotone in $H$, i.e., there exists a positive constant $C$ such that

$$
\begin{aligned}
& \left(L\left(v_{1}-v_{2}\right)+w_{1}-w_{2}, v_{1}-v_{2}\right) \geq C\left|v_{1}-v_{2}\right|^{2} \\
& \forall v_{1}, v_{2} \in D(B), \quad \forall w_{1} \in B v_{1}, \forall w_{2} \in B v_{2} .
\end{aligned}
$$

Let us finally specify suitable hypotheses on right hand side and initial data of the problem (1.1-2). We set

$$
\begin{gathered}
f=f_{1}+f_{2}, \quad f_{1} \in W^{2,1}\left(0, T ; V^{\prime}\right), \quad f_{2} \in H^{1}(0, T ; H), \\
u_{0} \in V, \quad v_{0} \in D(B),
\end{gathered}
$$

and prescribe the following compatibility condition

$$
\exists w_{0} \in B v_{0}: \quad f(0)-w_{0}-A u_{0} \in D\left(\varphi^{*}\right),
$$

where $D\left(\varphi^{*}\right)$ denotes the effective domain of the function $\left.\left.\varphi^{*}: H \rightarrow\right]-\infty,+\infty\right]$,

$$
\varphi^{*}(v):=\sup _{z \in H}\{(v, z)-\varphi(z)\}
$$

convex conjugate of the function $\varphi$ defined by (2.7) (see, e.g., [4] or [1] for information on convex functions and maximal monotone operators).

In the sequel we use the symbol " $"$ " to denote the time derivative of functions. Then the initial value problem can be stated as follows.

PROBLEM (P). Find $u \in H^{2}(0, T ; H) \cap W^{1, \infty}(0, T ; V)$ satisfying

$$
L u^{\prime \prime}(t)+w(t)+A u(t)=f(t)
$$

for some

$$
w(t) \in B u^{\prime}(t)
$$

a.e. with respect to $t \in] 0, T[$, and

$$
u(0)=u_{0}, \quad u^{\prime}(0)=v_{0}
$$

REMARK 1. Obviously the equation (2.16) has a meaning in $V^{\prime}$ and it can be equivalently rewritten as (cf. (2.6) and (2.9))

$$
\ell\left(u^{\prime \prime}(t), v\right)+(w(t), v)+a(u(t), v)=(f(t), v) \quad \forall v \in V .
$$

Note that a comparison of the terms in (2.16) (see also (2.12)) yields $w \in W^{1, \infty}\left(0, T ; V^{\prime}\right)+$ $L^{2}(0, T ; H)$. Actually, we will show that $L u^{\prime \prime} \in L^{\infty}(0, T ; H)$, whence $w \in L^{\infty}\left(0, T ; V^{\prime}\right)$.

THEOREM 1. There exists one and only one solution $u$ of Problem (P). Moreover $\Lambda u \in$ $W^{2, \infty}(0, T ; H)$. 
The existence of a solution to Problem (P) will be proved in the next sections by discretizing the problem and then passing to the limit in the finite difference scheme. Concerning the uniqueness, we are going to deduce it by contradiction. Let $u_{1}, u_{2}$ be two solutions of Problem (P) and let $w_{\imath}=f-L u_{\imath}^{\prime \prime}-A u_{\imath}, i=1,2$. Taking the difference of the respective equations (2.16), adding to both sides the term $L\left(u_{1}^{\prime}-u_{2}^{\prime}\right)+u_{1}-u_{2}$, multiplying the resulting equality by $u_{1}^{\prime}-u_{2}^{\prime}$, then integrating from 0 to $t \in[0, T]$, with the help of (2.18), (2.6), (2.9), and (2.4) we obtain

$$
\begin{aligned}
& \frac{1}{2} \ell\left(U^{\prime}(t), U^{\prime}(t)\right)+\int_{0}^{t}\left(L U^{\prime}(s)+W(s), U^{\prime}(s)\right) d s \\
& +\frac{1}{2} a_{1}(U(t), U(t))=\int_{0}^{t}\left(L U^{\prime}(s)+U(s), U^{\prime}(s)\right) d s
\end{aligned}
$$

where $U=u_{1}-u_{2}$ and $W=w_{1}-w_{2}$. Since $w_{\imath}(s) \in B u_{\imath}^{\prime}(s)$ for a.e. $\left.s \in\right] 0, T[, i=1,2$, recalling (2.8), (2.11), (2.5) and using the elementary inequality

$$
\lambda \mu \leq(\varepsilon / 2) \lambda^{2}+\mu^{2} /(2 \varepsilon) \quad \forall \lambda, \mu \in \mathbf{R}, \forall \varepsilon>0,
$$

from (2.19) it is not difficult to get

$$
\begin{aligned}
& \frac{1}{2}\left|\Lambda U^{\prime}(t)\right|^{2}+\frac{C}{2} \int_{0}^{t}\left|U^{\prime}(s)\right|^{2} d s+\frac{\omega_{1}}{2}\|U(t)\|^{2} \\
& \leq \int_{0}^{t}\left|\Lambda U^{\prime}(s)\right|^{2} d s+\frac{1}{2 C} \int_{0}^{t}|U(s)|^{2} d s \quad \forall t \in[0, T] .
\end{aligned}
$$

Owing to (2.21), there is a constant $C_{1}$ such that

$$
\left|\Lambda U^{\prime}(t)\right|^{2}+\|U(t)\|^{2} \leq C_{1} \int_{0}^{t}\left(\left|\Lambda U^{\prime}(s)\right|^{2}+\|U(s)\|^{2}\right) d s \quad \forall t \in[0, T]
$$

where, for instance, $C_{1}=\max \left\{2, C_{V} / C\right\} / \min \left\{1, \omega_{1}\right\}$ for some constant $C_{V}$ fulfilling

$$
|v| \leq C_{V}\|v\|^{2} \quad \forall v \in V .
$$

Then, by (2.22) and the Gronwall lemma we infer that $\|U(t)\|=0$ for any $t \in[0, T]$, which yields $u_{1}=u_{2}$. Thus, we have shown that Problem (P) has at most one solution.

Let us conclude the section by presenting, like applications of our abstract result, two simple examples of initial boundary value problems, for a partial differential equation and a system. In what follows, $\Omega \subset \mathbf{R}^{N}(N \geq 1)$ denotes a bounded domain with boundary $\Gamma$ (of class $C^{0,1}$, for instance) and outward normal $\nu=\left(\nu_{1}, \ldots, \nu_{N}\right)$ (defined a.e. in $\Gamma$ ). As usual, the variable in $\Omega \cup \Gamma$ is indicated by $x=\left(x_{1}, \ldots, x_{N}\right)$ and div, $\nabla, \Delta$ stand for the respective space differential operators divergence, gradient, Laplacian. In addition, for the sake of convenience we set $Q=\Omega \times] 0, T[$ and $\Sigma=\Gamma \times] 0, T[$.

EXAMPLE 1. The differential problem consists in finding a function $u: \bar{Q} \rightarrow \mathbf{R}$ which satisfies

$$
\begin{cases}h_{\ell}(x) u_{t t}+\beta_{\Omega}\left(x, u_{t}\right)-\operatorname{div}\left(h_{b}(x) \nabla u_{t}+h_{a}(x) \nabla u\right)=G(x, t) & \text { in } Q \\ \beta_{\Gamma}\left(x, u_{t}\right)+\left(h_{b} \nabla u_{t}+h_{a} \nabla u\right) \cdot \nu=g(x, t) & \text { on } \Sigma \\ u(x, 0)=u_{0}(x), \quad u_{t}(x, 0)=v_{0}(x) & x \in \Omega\end{cases}
$$

where $h_{\ell}, h_{b}, h_{a} \in L^{\infty}(\Omega)$ represent non negative coefficients, $\beta_{\Omega}: \Omega \times \mathbf{R} \rightarrow \mathbf{R}$ and $\beta_{\Gamma}:$ $\Gamma \times \mathbf{R} \rightarrow \mathbf{R}$ are Carathéodory functions (i.e., $\beta_{\Omega}(x, \xi)$ and $\beta_{\Gamma}(x, \xi)$ measurable in $x$ for any 
$\xi \in \mathbf{R}$ and continuous in $\xi$ for a.e. $x$ in $\Omega$ or $\Gamma$, respectively), and $G \in H^{1}\left(0, T ; L^{2}(\Omega)\right)$, $g \in W^{2,1}\left(0, T ; L^{2}(\Gamma)\right), u_{0}, v_{0} \in H^{1}(\Omega)$ complete the data. Moreover, assume there are three positive constants $c_{1}, c_{2}, c_{3}$ such that for any $\xi, \eta \in \mathbf{R}$ one has

$$
\begin{gathered}
h_{a}(x) \geq c_{1} \quad \text { for a.e. } x \in \Omega, \\
\left|\beta_{\Omega}(\cdot, \xi)\right| \leq c_{2}(|\xi|+1), \quad\left(\beta_{\Omega}(\cdot, \xi)-\beta_{\Omega}(\cdot, \eta)\right)(\xi-\eta) \geq 0 \quad \text { a.e. in } \Omega, \\
\left|\beta_{\Gamma}(\cdot, \xi)\right| \leq c_{2}(|\xi|+1), \quad\left(\beta_{\Gamma}(\cdot, \xi)-\beta_{\Gamma}(\cdot, \eta)\right)(\xi-\eta) \geq 0 \quad \text { a.e. in } \Gamma, \\
\left|h_{\ell}(x)(\xi-\eta)+\beta_{\Omega}(x, \xi)-\beta_{\Omega}(x, \eta)\right| \geq c_{3}|\xi-\eta| \geq 0 \quad \text { for a.e. } x \in \Omega .
\end{gathered}
$$

Thus, choosing $V=H^{1}(\Omega), H=L^{2}(\Omega), \ell(v, z)=\int_{\Omega} h_{\ell} v z, a(v, z)=\int_{\Omega} h_{a} \nabla v \cdot \nabla z, \quad D(B)=$ $H^{1}(\Omega)$ and

$$
(B v, z)=\int_{\Omega}\left(\beta_{\Omega}(\cdot, v) z+h_{b} \nabla v \cdot \nabla z\right)+\int_{\Gamma} \beta_{\Gamma}(\cdot, v) z
$$

for any $v, z \in H^{1}(\Omega)$, and identifying $f_{1}$ and $f_{2}$ with $g$ and $G$, respectively, it is a standard matter to see that (2.1-5) and (2.10-13) are fulfilled. Actually, the operator $B$ turns out monotone and demicontinuous from $H^{1}(\Omega)$ to $\left(H^{1}(\Omega)\right)^{\prime}$ (cf., e.g., [1, pp. 48-50]). Here, the condition (2.14) reduces to the existence of $z_{0} \in L^{2}(\Omega)$ such that

$$
\begin{aligned}
& \int_{\Omega}\left(G(\cdot, 0) z-\beta_{\Omega}\left(\cdot, v_{0}\right) z-\left(h_{b} \nabla v_{0}+h_{a} \nabla u_{0}\right) \cdot \nabla z\right) \\
& +\int_{\Gamma}\left(g(\cdot, 0)-\beta_{\Gamma}\left(\cdot, v_{0}\right)\right) z=\int_{\Omega} \sqrt{h_{\ell}} z_{0} z \quad \forall z \in H^{1}(\Omega) .
\end{aligned}
$$

Then we are allowed to apply Theorem 1 and conclude that the solution $u$ of the related Problem (P) lies in $H^{2}\left(0, T ; L^{2}(\Omega)\right) \cap W^{1, \infty}\left(0, T ; H^{1}(\Omega)\right)$. Note that $u$ is a weak solution of (2.24). Indeed, multiplying (2.16) by a test function $z \in H_{0}^{1}(\Omega)$ and integrating by parts, one concludes that

$$
\left.h_{\ell} u_{t t}+\beta_{\Omega}\left(\cdot, u_{t}\right)-\operatorname{div}\left(h_{b} \nabla u_{t}+h_{a} \nabla u\right)=G(\cdot, t) \quad \text { in } H^{-1}(\Omega) \text {, for a.e. } t \in\right] 0, T[.
$$

A subsequent comparison of the terms yields $\operatorname{div}\left(h_{b} \nabla u_{t}+h_{a} \nabla u\right) \in L^{\infty}\left(0, T ; L^{2}(\Omega)\right)$. Therefore, one can easily recover the boundary equality in (2.24) which, thanks to the hypotheses on $\beta_{\Gamma}$ and $g$, holds even in $L^{\infty}\left(0, T ; L^{2}(\Gamma)\right)$.

EXAMPLE 2. Let $\mathbf{b}$ denote a maximal monotone graph of $\mathbf{R}^{2} \times \mathbf{R}^{2}$, such that its domain contains $\mathbf{0}=(0,0)$ and $\mathbf{0} \in \mathbf{b}(\mathbf{0})$. Now, we look for generalized solutions $\mathbf{u}=\left(u_{1}, u_{2}\right): \bar{Q} \rightarrow \mathbf{R}^{2}$ of the following system

$$
\begin{cases}\left(u_{1}\right)_{t t}+w_{1}-\alpha_{1} \Delta u_{1}=F_{1}(x, t) & \text { in } Q \\ w_{2}-\alpha_{2} \Delta u_{2}=F_{2}(x, t) & \text { in } Q \\ \mathbf{w}=\left(w_{1}, w_{2}\right) \in \mathbf{b}\left(\mathbf{u}_{t}\right) & \text { in } Q \\ \mathbf{u}=\mathbf{0} & \text { on } \Sigma \\ \mathbf{u}(x, 0)=\mathbf{u}_{0}(x), \quad \mathbf{u}_{t}(x, 0)=\mathbf{v}_{0}(x) & x \in \Omega\end{cases}
$$

where $\alpha_{1}, \alpha_{2}$ are positive parameters, $\mathbf{F}=\left(F_{1}, F_{2}\right) \in H^{1}\left(0, T ;\left(L^{2}(\Omega)\right)^{2}\right)$, both $\mathbf{u}_{0}$ and $\mathbf{u}_{0}$ stay in $\left(H_{0}^{1}(\Omega)\right)^{2}$. Setting $V=\left(H_{0}^{1}(\Omega)\right)^{2}, H=\left(L^{2}(\Omega)\right)^{2}$, it is not difficult to verify that $\mathbf{b}$ induces a maximal monotone operator $B$ from $V$ to $V^{\prime}=\left(H^{-1}(\Omega)\right)^{2}$. The point is that, while $\mathbf{z} \in \mathbf{b}(\mathbf{v})$ a.e. in $Q$ whenever $\mathbf{z} \in(B \mathbf{v}) \cap H$, in general an element $\mathbf{z} \in B(\mathbf{v})$ has no meaning almost everywhere. Thus the relationship $\mathbf{w} \in \mathbf{b}\left(\mathbf{u}_{t}\right)$ in (2.25) must be understood in a generalized sense (this is the reason why we speak of $\mathbf{u}$ as a generalized solution). The definition of $L$ and 
$A$ being quite obvious in view of (2.25), we point out that here the validity of (2.5) extends to $\alpha=0$ and that the datum $\mathbf{F}$ plays as $f=f_{2}$ in (2.12). The further assumptions (2.11) and (2.13-14) of Theorem 1 are ensured by additional restrictions on $\mathbf{b}, \mathbf{u}_{0}, \mathbf{v}_{0}$, namely,

$$
\begin{gathered}
\text { the graph }\left(\xi_{1}, \xi_{2}\right) \mapsto\left(\xi_{1}, 0\right)+\mathbf{b}\left(\xi_{1}, \xi_{2}\right) \text { is strongly monotone, } \\
\mathbf{u}_{0}=\left(u_{01}, u_{02}\right) \in\left(H^{2}(\Omega)\right)^{2}, \quad \mathbf{v}_{0} \in D(B), \\
\exists \mathbf{w}_{0}=\left(w_{01}, w_{02}\right) \in\left(B \mathbf{v}_{0}\right) \cap\left(L^{2}(\Omega)\right)^{2}: \quad w_{02}=F(\cdot, 0)+\alpha_{2} \Delta u_{02} \quad \text { a.e. in } \Omega .
\end{gathered}
$$

Hence, thanks to Theorem 1 there exists a unique solution of the problem corresponding to (2.25). In addition, such solution satisfies the equations of (2.25) almost everywhere in $Q$ provided that, for instance, $\mathbf{b}$ is linearly bounded.

\section{APPROXIMATION.}

For the approximation of Problem (P) we introduce a backward finite differences scheme where, $n$ being an arbitrary positive integer, $\tau:=T / n$ denotes the time step. Since the functions $f_{1}, f_{2}$ are continuous from $[0, T]$ to $V^{\prime}$ (cf. (2.12)), for $i=0,1, \ldots, n$ we can set

$$
f_{1}^{i}:=f_{1}(\tau i), \quad f_{2}^{i}:=f_{2}(\tau i), \quad f^{2}:=f_{1}^{i}+f_{2}^{i} \in V^{\prime} .
$$

Then the approximating problem is formulated as follows.

PROBLEM $\left(\mathbf{P}_{\tau}\right)$. Find three vectors $\left(u^{0}, u^{1}, \ldots, u^{n}\right) \in V^{n+1},\left(v^{0}, v^{1}, \ldots, v^{n}\right) \in V^{n+1}$, $\left(w^{1}, \ldots, w^{n}\right) \in\left(V^{\prime}\right)^{n}$ such that

$$
u^{0}=u_{0}, \quad v^{0}=v_{0},
$$

and satisfying for $i=1, \ldots, n$

$$
\begin{gathered}
v^{2}=\frac{u^{i}-u^{i-1}}{\tau}, \\
w^{2} \in B v^{2}, \\
L \frac{v^{i}-v^{i-1}}{\tau}+w^{i}+A u^{2}=f^{2} .
\end{gathered}
$$

The next simple result states the existence and uniqueness of a discrete solution.

THEOREM 2. For any time step $\tau>0$ the Problem $\left(\mathrm{P}_{\tau}\right)$ has one and only one solution.

PROOF. As the operators $L$ and $A$ are linear, thanks to $(3.2-4)$ it suffices to prove that for any $g \in V^{\prime}$ there is one and only one $v \in V$ solving the equation

$$
\tau^{-1} L v+B v+\tau A v \ni g .
$$

Now, the mapping $\tau^{-1} L+\tau A$ is monotone and continuous from $V$ to $V^{\prime}$. Hence it results that (see, e.g., [1, Corollary 1.3, p. 48]) $\tau^{-1} L+B+\tau A$ is maximal monotone from $V$ to $V^{\prime}$. Also, on account of (2.11), (2.9), and (2.5), this operator is strongly monotone. Indeed, since by (2.11) we have that

$$
\tau^{-1}\left(L\left(v_{1}-v_{2}\right), v_{1}-v_{2}\right)+\left(\eta_{1}-\eta_{2}, v_{1}-v_{2}\right) \geq C \min \left\{1, \tau^{-1}\right\}\left|v_{1}-v_{2}\right|^{2}
$$

for any $v_{1}, v_{2} \in D(B), \eta_{1} \in B v_{1}, \eta_{2} \in B v_{2}$, then the above assertion follows from a subsequent application of (2.5). Therefore, the operator $\tau^{-1} L+B+\tau A$ turns out to be coercive, so that its range coincides with $V^{\prime}$ and the equation (3.6) has solutions. Moreover, the strong monotonicity also implies that there is a unique solution $v \in D(B)$. 
Before specifying the approximating functions useful for the sequel, let us introduce the auxiliary vector $\left(z^{0}, z^{1}, \ldots, \dot{z}^{n}\right) \in V^{n+1}$ defined by

$$
z^{0}=z^{1}, \quad z^{2}=\frac{v^{2}-v^{2-1}}{\tau}
$$

for $i=1, \ldots, n$. Then let $u_{\tau}, v_{\tau}, z_{\tau} \in L^{1}(0, T ; V)$ and $w_{\tau}, f_{\tau} \in L^{1}\left(0, T ; V^{\prime}\right)$ denote the piecewise constant functions determined by

$$
\begin{gathered}
u_{\tau}(t):=u^{2}, \quad v_{\tau}(t):=v^{2}, \quad z_{\tau}(t):=z^{2}, \\
w_{\tau}(t):=w^{2}, \quad f_{\tau}(t):=f^{2} \quad \text { if }(i-1) \tau<t \leq i \tau,
\end{gathered}
$$

for $i=1, \ldots, n$. We also consider the piecewise linear functions $\hat{u}_{\tau}, \hat{v}_{\tau}, \hat{z}_{\tau} \in W^{1,1}(0, T ; V)$ defined by (cf. (3.3) and (3.7))

$$
\begin{gathered}
\hat{u}_{\tau}(t):=u^{2}+v^{2}(t-i \tau), \quad \hat{v}_{\tau}(t):=v^{2}+z^{2}(t-i \tau), \\
\hat{z}_{\tau}(t):=z^{2}+\frac{z^{2}-z^{2-1}}{\tau}(t-i \tau) \quad \text { if }(i-1) \tau \leq t \leq i \tau,
\end{gathered}
$$

for $i=1, \ldots, n$. Observe that, in view of (3.8-9), the conditions and equations $(3.2-5)$ can be rewritten as

$$
\begin{gathered}
\hat{u}_{\tau}(0)=u_{0}, \quad \hat{v}_{\tau}(0)=v_{0}, \\
v_{\tau}(t)=\hat{u}_{\tau}^{\prime}(t), \\
w_{\tau}(t) \in B v_{\tau}(t), \\
L z_{\tau}(t)+w_{\tau}(t)+A u_{\tau}(t)=f_{\tau}(t)
\end{gathered}
$$

for a.e. $t \in] 0, T[$. Moreover, let us point out the further relationship

$$
z_{\tau}=\hat{v}_{\tau}^{\prime}
$$

Now, we are in a position to derive estimates, independent of $\tau$, for the discrete solutions.

\section{A PRIORI ESTIMATE.}

We start by writing the equation (3.5) also for the index $i-1$ and then take the difference. With the help of (3.3) and (3.7) it is straightforward to verify that

$$
L\left(z^{i}-z^{i-1}\right)+\tau L z^{i}+w^{i}-w^{i-1}+\tau\left(v^{i}+A v^{i}\right)=f^{i}-f^{i-1}+\tau L z^{i}+\tau v^{i}
$$

for $i=2, \ldots, n$. Multiplying (4.1) by $z^{2} \in V$ and recalling (2.6-7), (2.9), (2.4), we easily obtain

$$
\begin{aligned}
& \varphi\left(z^{i}\right)-\varphi\left(z^{i-1}\right)+\varphi\left(z^{i}-z^{i-1}\right)+\frac{1}{\tau}\left(L\left(v^{2}-v^{i-1}\right)+w^{2}-w^{2-1}, v^{2}-v^{i-1}\right) \\
& +\frac{1}{2} a_{1}\left(v^{i}, v^{2}\right)-\frac{1}{2} a_{1}\left(v^{i-1}, v^{i-1}\right)+\frac{\tau^{2}}{2} a_{1}\left(z^{2}, z^{2}\right)=\left(f^{i}-f^{z-1}+\tau L z^{i}+\tau v^{2}, z^{i}\right)
\end{aligned}
$$

for $i=2, \ldots, n$. Next, we sum (4.2) with respect to $i$. Setting

$$
\begin{aligned}
S_{m}:=\varphi\left(z^{m}\right) & +\sum_{i=1}^{m} \varphi\left(z^{z}-z^{i-1}\right)+\sum_{i=1}^{m} \cdot \frac{1}{\tau}\left(L\left(v^{i}-v^{i-1}\right)+w^{2}-w^{i-1}, v^{i}-v^{i-1}\right) \\
& +\frac{1}{2} a_{1}\left(v^{m}, v^{m}\right)+\sum_{i=1}^{m} \frac{\tau^{2}}{2} a_{1}\left(z^{i}, z^{2}\right)
\end{aligned}
$$


for $m=1, \ldots, n$, where $w^{0}:=w_{0}$ is defined in (2.14), it is not difficult to see that

$$
S_{m}=S_{1}+\sum_{i=2}^{m} \tau\left(\frac{f^{2}-f^{i-1}}{\tau}+L z^{2}+v^{2}, z^{i}\right)
$$

for $m=2, \ldots, n$. Consider now the equality $(3.5)$ for $\imath=1$ and multiply it by $z^{1}$. Thanks to (3.7), (3.2-3), (2.6-7), (2.9), (2.4), and (3.1), we easily infer that

$$
\varphi\left(z^{1}\right)+S_{1}=\left(f(0)-w_{0}-A u_{0}, z^{1}\right)+\frac{1}{2} a_{1}\left(v_{0}, v_{0}\right)+\tau\left(\frac{f^{1}-f^{0}}{\tau}+L z^{1}+v^{1}, z^{1}\right) .
$$

As $\left(f(0)-w_{0}-A u_{0}, z^{1}\right)-\varphi\left(z^{1}\right) \leq \varphi^{*}\left(f(0)-w_{0}-A u_{0}\right)$ because of (2.14-15), from (4.4-5) it follows that

$$
S_{m} \leq C_{2}+\sum_{i=1}^{m} \tau\left(\frac{f^{\imath}-f^{i-1}}{\tau}+L z^{2}+v^{2}, z^{2}\right)
$$

for $m=1, \ldots, n$, where $C_{2}=\varphi^{*}\left(f(0)-w_{0}-A u_{0}\right)+a_{1}\left(v_{0}, v_{0}\right) / 2$ (see also (2.13)). But, taking (2.7-8), (2.11), (3.7), and (2.5) into account, we have that

$$
\begin{aligned}
S_{m} \geq N_{m} & :=\frac{1}{2}\left|\Lambda z^{m}\right|^{2}+\frac{\tau}{2} \sum_{i=1}^{m} \tau\left|\Lambda \frac{z^{i}-z^{i-1}}{\tau}\right|^{2} \\
& +C \sum_{i=1}^{m} \tau\left|z^{i}\right|^{2}+\frac{\omega_{1}}{2}\left\|v^{m}\right\|^{2}+\frac{\omega_{1} \tau}{2} \sum_{i=1}^{m} \tau\left\|z^{i}\right\|^{2} .
\end{aligned}
$$

We want to estimate the right hand side of (4.6) with respect to $N_{m}$. Setting (cf. (3.1))

$$
g_{1}^{i}:=\frac{f_{1}^{2}-f_{1}^{2-1}}{\tau}, \quad g_{2}^{2}:=\frac{f_{2}^{2}-f_{2}^{i-1}}{\tau}
$$

for $i=1, \ldots, n$, by $(4.6-7),(3.2),(3.7),(2.6)$, and (2.8) it is not difficult to check that

$$
N_{m} \leq C_{2}+\sum_{j=1}^{4} R_{\jmath}(m)
$$

where

$$
\begin{gathered}
R_{1}(m):=\left(g_{1}^{m}, v^{m}\right)-\left(g_{1}^{1}, v^{0}\right)-\sum_{i=2}^{m} \tau\left(\frac{g_{1}^{i}-g_{1}^{i-1}}{\tau}, v^{i-1}\right) \\
R_{2}(m):=\sum_{i=1}^{m} \tau\left|g_{2}^{i}\right|\left|z^{i}\right|, \quad R_{3}(m):=\sum_{i=1}^{m} \tau\left|\Lambda z^{i}\right|^{2}, \quad R_{4}(m):=\sum_{i=1}^{m} \tau\left|v^{i}\right|\left|z^{i}\right|,
\end{gathered}
$$

for $m=1, \ldots, n$. Noting that (see (4.8), (3.1), and (2.12))

$$
\begin{aligned}
\left\|g_{1}^{j}\right\|_{*} & \leq \frac{1}{\tau} \int_{(j-1) \tau}^{j \tau}\left\|f_{1}^{\prime}(t)\right\|_{*} d t \leq\left\|f_{1}^{\prime}\right\|_{C^{0}\left([0, T] ; V^{\prime}\right)}, \\
\left\|\frac{g_{1}^{i}-g_{1}^{2-1}}{\tau}\right\|_{*} & \leq \frac{1}{\tau^{2}}\left\|\int_{(i-1) \tau}^{\imath \tau}\left(f_{1}^{\prime}(t)-f_{1}^{\prime}(t-\tau)\right) d t\right\|_{*} \\
& \leq \frac{1}{\tau^{2}} \int_{(\imath-1) \tau}^{\imath \tau} \int_{t-\tau}^{t}\left\|f_{1}^{\prime \prime}(s)\right\|_{*} d s d t \leq \frac{1}{\tau}\left\|f_{1}^{\prime \prime}\right\|_{L^{1}\left((i-2) \tau, \imath \tau ; V^{\prime}\right)}
\end{aligned}
$$

for $j=1, \ldots, n$ and $i=2, \ldots, n$, by using (2.20) one can easily deduce that there is a constant $C_{3}$ fulfilling

$$
\left|R_{1}(m)\right| \leq C_{3}+\frac{\omega_{1}}{8} \max _{1 \leq j \leq m}\left\|v^{j}\right\|^{2}
$$


with, for instance,

Since

$$
C_{3}=\frac{8}{\omega_{1}}\left\|f_{1}^{\prime}\right\|_{C^{0}\left([0, T] ; V^{\prime}\right)}^{2}+\frac{\omega_{1}}{16}\left\|v_{0}\right\|^{2}+\frac{16}{\omega_{1}}\left\|f_{1}^{\prime \prime}\right\|_{L^{1}\left(0, T ; V^{\prime}\right)}^{2} .
$$

$$
\left|g_{2}^{2}\right|^{2} \leq \frac{1}{\tau}\left\|f_{2}^{\prime}\right\|_{L^{2}((\imath-1) \tau, \imath \tau ; H)}^{2}
$$

owing to the Hölder inequality (cf. also (2.12)), another application of (2.20) yields

$$
R_{2}(m) \leq \frac{1}{C}\left\|f_{2}^{\prime}\right\|_{L^{2}(0, T ; H)}^{2}+\frac{C}{4} \sum_{i=1}^{m} \tau\left|z^{2}\right|^{2} .
$$

Letting the term $R_{3}(m)$ in its present form, for the estimate of $R_{4}$ we recall $(2.23)$ and infer that

$$
R_{4}(m) \leq \frac{C_{V}}{C} \sum_{i=1}^{m} \tau\left\|v^{\imath}\right\|^{2}+\frac{C}{4} \sum_{i=1}^{m} \tau\left|z^{\imath}\right|^{2} .
$$

Then, in view of (4.7) and (4.9-12), it is straightforward to calculate a constant $C_{4}$, independent of $\tau$, such that

$$
\frac{1}{2} N_{k} \leq C_{4}\left(1+\sum_{i=1}^{m} \tau N_{i}\right)+\frac{1}{4} \max _{1 \leq j \leq m} N
$$

for any $k, m$ satisfying $1 \leq k \leq m \leq n$. Hence, taking the maximum with respect to $k$, we obtain

$$
\frac{1}{4} N_{m} \leq \frac{1}{4} \max _{1 \leq j \leq m} N_{j} \leq C_{4}\left(1+\sum_{i=1}^{m} \tau N_{i}\right)
$$

Setting now $\bar{\tau}=1 /\left(8 C_{4}\right)$, as $N_{m} \geq 0$ we have that

$$
N_{1} \leq 8 C_{4}, \quad N_{m} \leq 8 C_{4}\left(1+\sum_{i=1}^{m-1} \tau N_{i}\right)
$$

if $m=2, \ldots, n$, for any time step $\tau=T / n<\bar{\tau}$. By applying the discrete Gronwall lemma to the finite sequences $\left\{N_{1}, \ldots, N_{n}\right\}$, from (4.13) we conclude, for instance, that

$$
N_{m} \leq 8 C_{4} \exp \left(8 C_{4} T\right)
$$

for $m=1, \ldots, n$. Therefore, on account of the definitions (4.7) and (3.8-9), there is a constant $C_{5}$ such that for any time step $\tau<\bar{\tau}$ one has

$$
\begin{array}{r}
\left\|\Lambda z_{\tau}\right\|_{L^{\infty}(0, T ; H)}^{2}+\tau\left\|\Lambda \hat{z}_{\tau}^{\prime}\right\|_{L^{2}(0, T ; H)}^{2}+\left\|z_{\tau}\right\|_{L^{2}(0, T ; H)}^{2} \\
+\left\|v_{\tau}\right\|_{L^{\infty}(0, T ; V)}^{2}+\tau\left\|z_{\tau}\right\|_{L^{2}(0, T ; V)}^{2} \leq C_{5} .
\end{array}
$$

REMARK 2. Let us point out that the bound $\bar{\tau}$ only depends on the quantities $a_{1}\left(v_{0}, v_{0}\right)$, $\varphi^{*}\left(f(0)-w_{0}-A u_{0}\right),\left\|f_{1}^{\prime}\right\|_{W^{1,1}\left(0, T ; V^{\prime}\right)},\left\|f_{2}^{\prime}\right\|_{L^{2}(0, T ; H)}, C_{V}, \omega_{1}$, and $C$. The same holds for the constant $C_{5}$, which depends upon $T$ as well.

Owing to (4.14) along with (3.8-11), (2.13), (3.14), we easily deduce the further estimate

$$
\left\|\hat{v}_{\tau}\right\|_{H^{1}(0, T ; H) \cap L^{\infty}(0, T ; V)}^{2}+\left\|\hat{u}_{\tau}\right\|_{W^{1, \infty}(0, T ; V)}^{2} \leq C_{6}
$$

for some constant $C_{6}$ depending only on $C_{5},\left\|u_{0}\right\|$, and $\left|v_{0}\right|$. As $L=\Lambda^{2}$ (cf. (2.6), (2.8), and (2.1)) note that (4.14) also yields

$$
\left\|L z_{\tau}\right\|_{L^{\infty}(0, T ; H)}^{2} \leq C_{\ell} C_{5}
$$

Hence, with the help of (3.8), (3.1), (2.12), and of a comparison in (3.13), it results that

$$
\left\|w_{\tau}\right\|_{L^{\infty}\left(0, T ; V^{\prime}\right)}^{2} \text { is bounded independently of } \tau \text {. }
$$

Thanks to (4.14-17), taking the limit in Problem $\left(\mathrm{P}_{\tau}\right)$ as $\tau$ tends to 0 , we are able to find a solution of Problem (P), thus achieving the proof of Theorem 1. This argument will be developed in the next section. 


\section{PASSAge to the limit.}

Recalling that $\tau=T / n$, let us first state the following convergence property (see (3.8), (3.1), and (2.12))

$$
\left\|f_{\tau}-f\right\|_{L^{2}\left(0, T, V^{\prime}\right)}^{2}=\sum_{i=1}^{n} \int_{(i-1) \tau}^{i \tau}\left\|\int_{t}^{3 \tau} f^{\prime}(s) d s\right\|_{*}^{2} d t \leq \tau^{2}\left\|f^{\prime}\right\|_{L^{2}\left(0, T ; V^{\prime}\right)}^{2} .
$$

Then, owing to the estimates (4.14-17), there exist $z, v, u, w$ such that, possibly taking subsequences,

$$
\begin{aligned}
z_{\tau} \rightarrow z \text { weakly in } L^{2}(0, T ; H), & \\
\hat{v}_{\tau} \rightarrow v & \text { weakly star in } H^{1}(0, T ; H) \cap L^{\infty}(0, T ; V), \\
\hat{u}_{\tau} & \rightarrow u \text { weakly star in } W^{1, \infty}(0, T ; V), \\
L_{\tau} & \rightarrow L z \text { weakly star in } L^{\infty}(0, T ; H), \\
w_{\tau} & \rightarrow w \text { weakly star in } L^{\infty}\left(0, T ; V^{\prime}\right)
\end{aligned}
$$

as $\tau \searrow 0$. Since (cf. (3.8-9), (3.14), and (4.14))

$$
\left\|v_{\tau}-\hat{v}_{\tau}\right\|_{L^{\infty}(0, T ; H)}^{2} \leq \max _{1 \leq \imath \leq n} \tau^{2}\left|\frac{v^{2}-v^{z-1}}{\tau}\right|^{2} \leq \tau\left\|z_{\tau}\right\|_{L^{2}(0, T ; H)}^{2} \leq C_{5} \tau,
$$

by (5.3) and (4.14) we have that

$$
v_{\tau} \rightarrow v \text { weakly star in } L^{\infty}(0, T ; V)
$$

whence, due to (3.11), (3.14), and (5.2-4) as well,

$$
u^{\prime}=v, \quad v^{\prime}=z
$$

Therefore, it turns out that $u \in H^{2}(0, T ; H)$ too, and

$$
u^{\prime \prime}=z
$$

In order to show the existence of solutions of Problem (P) and thus complete the proof of Theorem 1, we establish the following result.

THEOREM 3. The above defined limit function $u$ solves Problem (P). Moreover, the convergences (5.2-6) hold not only for subsequences, but for the whole sequences.

PROOF. Noting that the last part of the statement follows easily from the uniqueness for the solution of Problem (P), let us just check that $u$, along with the auxiliary function $w$ specified by (5.6), satisfies (2.16-18). In view of (3.10), the initial conditions (2.18) result from (5.3-4) and (5.9). Next, observing that

$$
\left\|u_{\tau}-\hat{u}_{\tau}\right\|_{L^{\infty}(0, T ; V)}^{2} \leq \tau^{2}\left\|v_{\tau}\right\|_{L^{\infty}(0, T ; V)}^{2} \leq C_{5} \tau^{2}
$$

because of (3.8-9) and (4.14), it is straightforward to deduce that

$$
u_{\tau} \rightarrow u \text { weakly star in } L^{\infty}(0, T ; V)
$$


as $\tau$ goes to 0 . Hence, on account also of (5.5), (5.10), (5.6), (2.9), (2.3), and (5.1), passing to the limit in (3.13) we obtain (2.16) a.c. with respect to $t \in] 0, T[$. Then, it remains to show (2.17). To this aim, let us consider the following operator $\mathcal{B}$ from $L^{2}(0, T ; V)$ to $L^{2}\left(0, T ; V^{\prime}\right)$,

$$
y \in \mathcal{B}(x) \text { if and only if } y(t) \in B(x(t)) \text { for a.e. } t \in] 0, T[\text {. }
$$

By standard arguments (see, e.g., [4] or [1]) it is not difficult to verify that $\mathcal{B}$ is maximal monotone. Therefore, recalling (3.12), (5.6), (5.8-9), and Lemma 1.3 of [1, Chapter II], it suffices to prove that

$$
\underset{\tau \searrow 0}{\limsup } \int_{0}^{T}\left(w_{\tau}(t), v_{\tau}(t)\right) d t \leq \int_{0}^{T}\left(w(t), u^{\prime}(t)\right) d t .
$$

Now, thanks to (3.10-11), (3.13-14), (2.6), and (2.9) we have that

$$
\begin{aligned}
& \int_{0}^{T}\left(w_{\tau}(t), v_{\tau}(t)\right) d t=-\frac{1}{2} \ell\left(\hat{v}_{\tau}(T), \hat{v}_{\tau}(T)\right)+\frac{1}{2} \ell\left(v_{0}, v_{0}\right) \\
& +\int_{0}^{T} \ell\left(\hat{v}_{\tau}^{\prime}(t),\left(\hat{v}_{\tau}-v_{\tau}\right)(t)\right) d t-\frac{1}{2} a\left(\hat{u}_{\tau}(T), \hat{u}_{\tau}(T)\right) \\
& +\frac{1}{2} a\left(u_{0}, u_{0}\right)+\int_{0}^{T} a\left(\left(\hat{u}_{\tau}-u_{\tau}\right)(t), \hat{u}_{\tau}^{\prime}(t)\right) d t+\int_{0}^{T}\left(f_{\tau}(t), \hat{u}_{\tau}^{\prime}(t)\right) d t
\end{aligned}
$$

for $L$ and $A$ are selfadjoint operators. Then, the convergences (5.3-4) and (5.1), the estimates (5.7) and (5.11), and the lower semicontinuity of the quadratic functions specified by $\ell$ and $a$ (regarding both strong and weak topology of $H$ and $V$, respectively) allow us to infer that

$$
\begin{array}{r}
\underset{\tau \searrow 0}{\limsup } \int_{0}^{T}\left(w_{\tau}(t), v_{\tau}(t)\right) d t \leq-\frac{1}{2} \ell(v(T), v(T))+\frac{1}{2} \ell\left(v_{0}, v_{0}\right) \\
-\frac{1}{2} a(u(T), u(T))+\frac{1}{2} a\left(u_{0}, u_{0}\right)+\int_{0}^{T}\left(f(t), u^{\prime}(t)\right) d t .
\end{array}
$$

But, on account of (2.16) and (5.9), the right hand side of (5.15) turns out to coincide with $\int_{0}^{T}\left(w(t), u^{\prime}(t)\right) d t$, so that $(5.13)$ is completely proved.

\section{ERROR ESTIMATE.}

In this section we estimate the difference between the solution $u$ of Problem (P) and the function $\hat{u}_{\tau}$ introduced in (3.9). By our procedure we obtain a rate of convergence (of $\hat{u}_{\tau}$ to $u$ ) of order $\tau^{1 / 2}$ in suitable norms. Recalling that the constants $\bar{\tau}$ and $C_{5}$ are defined in Section 4 (see Remark 2), here we prove the following

THEOREM 4. There exists a constant $C_{7}$, depending only on $C, \omega_{1}, C_{5}, T, C_{V}, C_{a}$, and $\left\|f^{\prime}\right\|_{L^{2}\left(0, T ; V^{\prime}\right)}$, such that for any time step $\left.\tau \in\right] 0, \bar{\tau}[$ there holds

$$
\left\|\Lambda\left(u-\hat{u}_{\tau}\right)^{\prime}\right\|_{L^{\infty}(0, T ; H)}+\left\|u-\hat{u}_{\tau}\right\|_{H^{1}(0, T ; H) \cap C^{0}([0, T] ; V)} \leq C_{7} \tau^{1 / 2} .
$$

PROOF. Taking the difference between (2.16) and (3.13), with the help of (2.6), (2.9), (2.4), (3.11), and (3.14) one can easily verify that

$$
\begin{array}{r}
\ell\left(u^{\prime \prime}-\hat{v}_{\tau}^{\prime}, u^{\prime}-\hat{v}_{\tau}\right)+\left(L\left(u^{\prime}-v_{\tau}\right)+w-w_{\tau}, u^{\prime}-v_{\tau}\right)+a_{1}\left(u-\hat{u}_{\tau}, u^{\prime}-\hat{u}_{\tau}^{\prime}\right) \\
=\ell\left(u^{\prime}-v_{\tau}, u^{\prime}-v_{\tau}\right)+\ell\left(u^{\prime \prime}-z_{\tau}, v_{\tau}-\hat{v}_{\tau}\right)+\left(u-\hat{u}_{\tau}, u^{\prime}-v_{\tau}\right) \\
\left.+a\left(u_{\tau}-\hat{u}_{\tau}, u^{\prime}-v_{\tau}\right)+\left(f-f_{\tau}, u^{\prime}-v_{\tau}\right) \text { a.e. in }\right] 0, T[.
\end{array}
$$


Next, we integrate (6.2) from 0 to $t \in[0, T]$ accounting for the initial conditions (2.18) and (3.10). In view of $(2.8),(2.11),(2.5),(2.3),(2.12)$, and (3.8), it is not difficult to infer that

$$
N(t):=\frac{1}{2}\left|\Lambda\left(u^{\prime}-\hat{v}_{\tau}\right)(t)\right|^{2}+C \int_{0}^{t}\left|\left(u^{\prime}-v_{\tau}\right)(s)\right|^{2} d s+\frac{\omega_{1}}{2}\left\|\left(u-\hat{u}_{\tau}\right)(t)\right\|^{2} \leq \sum_{\jmath=1}^{6} Q_{\jmath}(t),
$$

where

$$
\begin{gathered}
Q_{1}(t):=\int_{0}^{t}\left|\Lambda\left(u^{\prime}-v_{\tau}\right)(s)\right|^{2} d s, \\
Q_{2}(t):=\int_{0}^{t}\left|\Lambda\left(u^{\prime \prime}-z_{\tau}\right)(s)\right|\left|\Lambda\left(v_{\tau}-\hat{v}_{\tau}\right)(s)\right| d s, \\
Q_{3}(t):=\int_{0}^{t}\left|\left(u-\hat{u}_{\tau}\right)(s)\right|\left|\left(u^{\prime}-v_{\tau}\right)(s)\right| d s, \\
Q_{4}(t):=C_{a} \int_{0}^{t}\left\|\left(u_{\tau}-\hat{u}_{\tau}\right)(s)\right\|\left\|\left(u^{\prime}-v_{\tau}\right)(s)\right\| d s, \\
Q_{5}(t):=\int_{0}^{t}\left\|\left(f-f_{\tau}\right)(s)\right\|_{*}\left\|\left(u^{\prime}-v_{\tau}\right)(s)\right\| d s,
\end{gathered}
$$

for any $t \in[0, T]$. Now, we estimate each one of these integrals. Since (cf. (3.8-9) and (4.14))

$$
\left\|\Lambda\left(\hat{v}_{\tau}-v_{\tau}\right)\right\|_{L^{\infty}(0, T ; H)}^{2} \leq \tau^{2}\left\|\Lambda z_{\tau}\right\|_{L^{\infty}(0, T ; H)}^{2} \leq C_{5} \tau^{2}
$$

it is straightforward to see that

$$
Q_{1}(t) \leq 2 \int_{0}^{t}\left|\Lambda\left(u^{\prime}-\hat{v}_{\tau}\right)(s)\right|^{2} d s+2 C_{5} T \tau^{2} .
$$

Thanks to (4.14), (5.2), and (5.10), we have that $\left\|\Lambda u^{\prime \prime}\right\|_{L^{\infty}(0, T ; H)} \leq C_{5}^{1 / 2}$. Hence, the following inequality

$$
Q_{2}(t) \leq 2 C_{5} T \tau
$$

results from (6.4). By (2.20) and (2.23) we deduce that

$$
Q_{3}(t) \leq \frac{C_{V}}{2 C} \int_{0}^{t}\left\|\left(u-\hat{u}_{\tau}\right)(s)\right\|^{2} d s+\frac{C}{2} \int_{0}^{t}\left|\left(u^{\prime}-v_{\tau}\right)(s)\right|^{2} d s .
$$

Recalling (5.11), (4.14) and arguing as in the estimate of $Q_{2}$, we obtain

$$
Q_{4}(t) \leq 2 C_{a} C_{5} T \tau
$$

Owing to (5.1) and to the Hölder inequality, similarly we have that

$$
Q_{5}(t) \leq 2\left(C_{5} T\right)^{1 / 2}\left\|f^{\prime}\right\|_{L^{2}\left(0, T ; V^{\prime}\right)} \tau .
$$

Therefore, due to (6.3) and (6.5-9), one can easily determine a constant $C_{8}$ such that

$$
N(t) \leq C_{8}\left(\tau+\int_{0}^{t} N(s) d s\right) \quad \forall t \in[0, T]
$$

where $C_{8}$ has the same dependences as $C_{7}$. By applying the Gronwall lemma to (6.10), we find out that

$$
N(t) \leq C_{8} \exp \left(C_{8} T\right) \tau \quad \forall t \in[0, T]
$$


and, thanks to the definition of $N$ coupled with (6.4) and (3.11), the last inequality implies (6.1). This concludes the proof of Theorem 4 .

REMARK 3. The rates of convergence in (6.1) may appear non optimal from the point of view of the numerical analysis, in regard to the regularity of the solution $u$ to Problem (P). But the order $\tau^{1 / 2}$ is optimal with respect to the hyperbolic nature of the problem (an interesting and rigorous discussion on the optimality, distinguishing between parabolic and hyperbolic Cauchy problems, is carried out in [12]), even in the simple case where $L=I$ (cf. [1, pp. 268-269]).

ACKNOWLEDGEMENT. This work has been partially supported by Istituto di Analisi Numerica del Consiglio Nazionale delle Ricerche, Via Abbiategrasso 209, 27100 Pavia, Italy. The authors would like to express their gratitude to the referee, who urged them to improve the contents of the paper.

\section{REFERENCES}

[1] BARBU, V. Nonlinear semigroups and differential equations in Banach spaces, Noordhoff International Publishing, Leyden, 1976.

[2] BENSOUSSAN, A., LIONS, J.L., PAPANICOLAU, G.C. Perturbations et "augmentation" des conditions initiales, Singular perturbations and boundary layer theory, Lecture Notes in Math. 594, Springer, Berlin, 1977, pp. 10-29.

[3] BRÉZIS, H. Semi-groupes non linéaires et applications, Sympos. Math. 7, Academic Press, London, 1971, pp. 3-27.

[4] BRÉZIS, H. Opérateurs maximaux monotones et semi-groupes de contractions dans les espaces de Hilbert, North-Holland, Amsterdam, 1973.

.[5] CARROLL, R.W., SHOWALTER, R.E. Singular and degenerate Cauchy problems, Math. Sci. Engrg. 27, Academic Press, New York, 1976.

[6] COLLI, P., FAVINI, A. On some degenerate second order equations of mixed type, Funkcial. Ekvac., to appear.

[7] DE LIMA, O.A. Existence and uniqueness of solutions for an abstract nonlinear hyperbolicparabolic equation, Appl. Anal. 24 (1987), 101-116.

[8] LIONS, J.L. Quelques méthodes de résolution des problèmes aux limites non linéaires, Dunod Gauthier-Villars, Paris, 1969.

[9] LIONS, J.L., STRAUSS, W.A. Some non-linear evolution equations, Bull. Soc. Math. France 9 (1965), 43-96.

[10] MACIEL, A.B. On hyperbolic-parabolic equation with a continuous nonlinearity, Nonlinear Anal. 20 (1993), 745-754.

[11] MEDEIROS, L.A. Non linear hyperbolic-parabolic partial differential equations, Funkcial. Ekvac. 23 (1980), 151-158.

[12] RULLA, J. Error analyses for implicit approximations to solutions to Cauchy problems, SIAM J. Numer. Anal., to appear. 


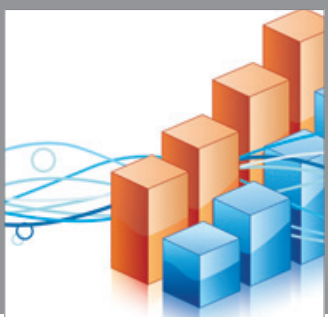

Advances in

Operations Research

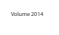

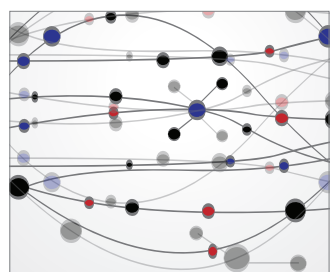

\section{The Scientific} World Journal
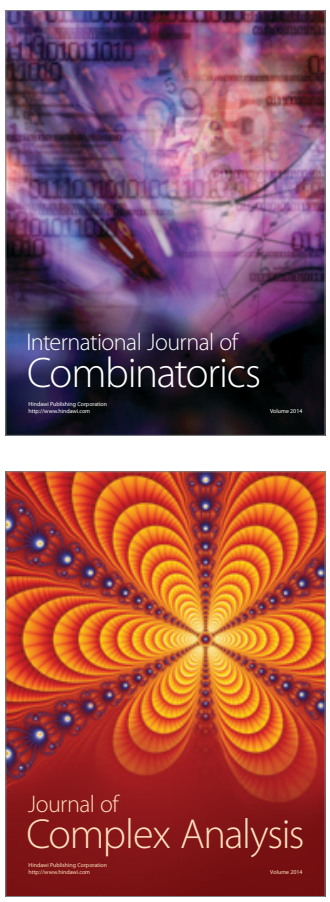

International Journal of

Mathematics and

Mathematical

Sciences
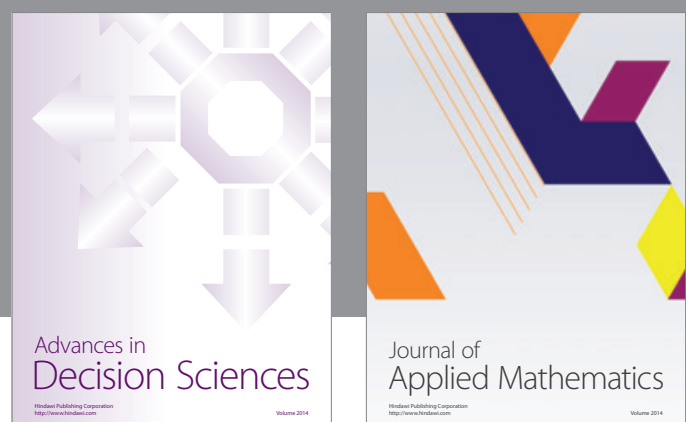

Journal of

Applied Mathematics
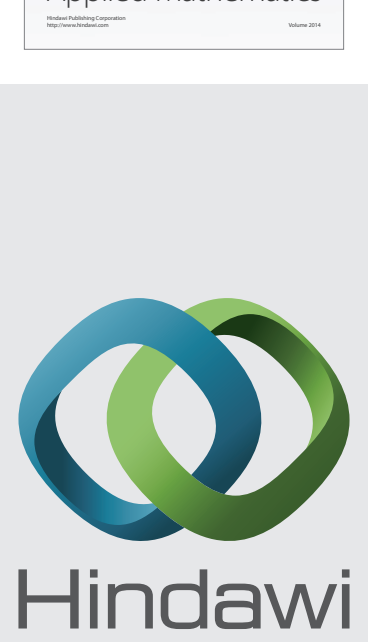

Submit your manuscripts at http://www.hindawi.com
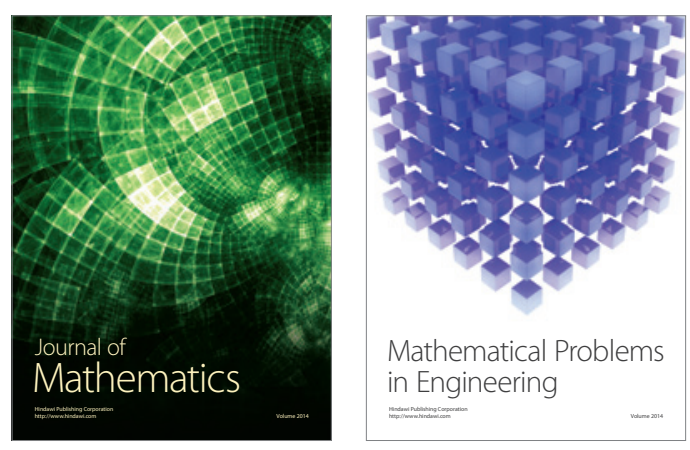

Mathematical Problems in Engineering
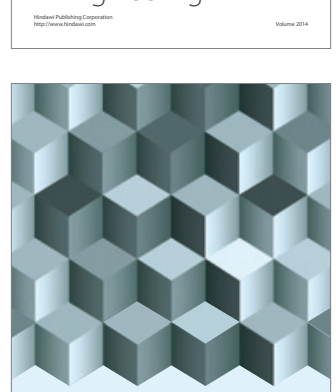

Journal of

Function Spaces
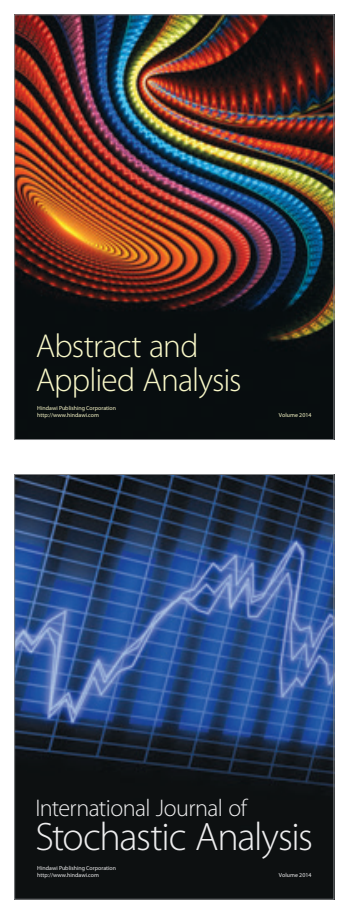

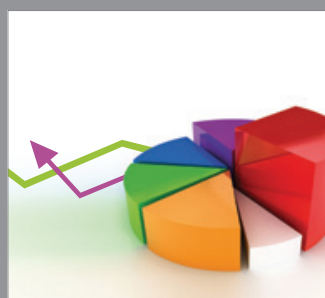

ournal of

Probability and Statistics

Promensencen
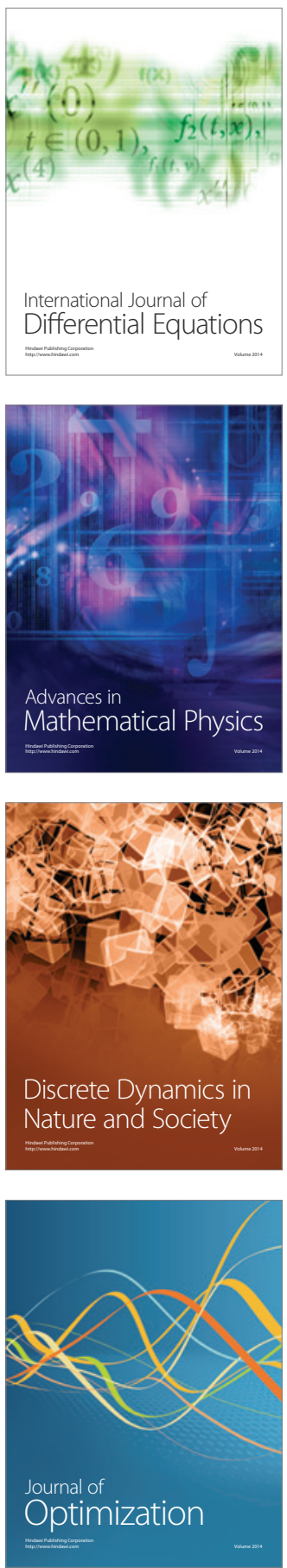